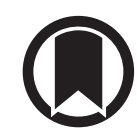

CrossMark

\title{
Continuous positive airway pressure in COVID-19 patients with moderate-to- severe respiratory failure
}

\author{
To the Editor:
}

Noninvasive ventilation (NIV) and continuous positive airway pressure (CPAP) are recommended for acute hypoxaemic respiratory failure (AHRF) due to cardiogenic pulmonary oedema but no recommendation has been made for viral pandemics, because of the lack of randomised studies showing their efficacy and concerns of infection dissemination [1]. Early after the coronavirus disease 2019 (COVID-19) outbreak in Italy, there was an expert consensus in favour of CPAP and NIV as first-line treatments for the associated AHRF [2]. However, few studies used unspecified NIV in a minority of patients without detailed results [3-5]. CPAP was the object of two short reports with differing results [6, 7]. Thus, their value in COVID-19 remains to be established.

2 weeks after the outbreak of COVID-19 in our region (Liguria, Italy), we systematically started using CPAP in patients with moderate-to-severe AHRF, following an ad hoc algorithm. Herein, we report the results obtained in all consecutive patients admitted during the first 4 weeks after the algorithm implementation. The primary outcome of the study was 4 -week survival without invasive mechanical ventilation (IMV).

We retrospectively reviewed the records of patients admitted to the COVID-19 unit of the 400-bed Galliera Hospital (Genoa, Italy) between 16 March and 12 April 2020. Two 20-bed general wards were adapted as sub-intensive units with a filter area for donning and doffing personal protective equipment including N-95 masks. The local ethics committee approved the study (n.5/2020) and waived written consent owing to the observational design.

Ground-glass bilateral pulmonary infiltrates on computed tomography (CT) and positive oropharyngeal swabs for severe acute respiratory-syndrome-coronavirus-2 confirmed COVID-19 pneumonia. Pharmacological treatment included 7-days of oral hydroxychloroquine (200 mg) plus methylprednisolone $\left(1 \mathrm{mg} \cdot \mathrm{kg}^{-1}\right)$ twice daily, followed by 7 -days of intravenous anakinra $(1300 \mathrm{mg})$ or single-dose tocilizumab $\left(8 \mathrm{mg} \cdot \mathrm{kg}^{-1}\right)$ in case of persistently high inflammatory markers.

Treatment strategy was initially based on arterial oxygen tension/inspiratory oxygen fraction $\left(P_{\mathrm{aO}_{2}} / F_{\mathrm{IO}_{2}}\right)$ on room air, breathing frequency $(\mathrm{BF})$ and presence of dyspnoea, and then adjusted following an ad hoc algorithm (figure 1a). Patients with oxygen saturation measured by pulse oximetry $<95 \%$ or $P_{\mathrm{aO}_{2}} / F_{\mathrm{IO}_{2}}>200$ received oxygen via Ventimask. Criteria for CPAP were $P_{\mathrm{aO}_{2}} / F_{\mathrm{IO}_{2}}<200, P_{\mathrm{aO}_{2}}<60 \mathrm{mmHg}$, breathing frequency $>30$ breaths $\cdot \mathrm{min}^{-1}$, dyspnoea at rest or during minimal efforts, if any. IMV was considered after 4 days of unsuccessful CPAP, i.e. with $P_{\mathrm{aO}_{2}} / F_{\mathrm{IO}_{2}}$ tending to decrease, breathing frequency $>30$ breaths $\cdot \mathrm{min}^{-1}$ and $P_{\mathrm{aO}_{2}}<60 \mathrm{mmHg}$.

Three types of Venturi generators were available (EasyVent, EasyFlow and CPAP Flow Generator with Monitor) and adapted to deliver outflows of $100-150 \mathrm{~L} \cdot \mathrm{min}^{-1}$ with anti-viral filters positioned to both inlet and outlet ports. CPAP was $10 \mathrm{cmH}_{2} \mathrm{O}$ in all patients and $F_{\mathrm{IO}_{2}}$ was $40-60 \%$ titrated to $P_{\mathrm{aO}_{2}} \geqslant 60 \mathrm{mmHg}$. During CPAP, patients were in a semi-supine or sitting position. Weaning was started when no desaturation, tachypnoea or tachycardia appeared during CPAP interruptions for eating with $P_{\mathrm{aO}_{2}} / F_{\mathrm{IO}_{2}}>250$ and tending to increase for at

@ERSpublications

CPAP can be successfully used in a number of COVID-19 patients with hypoxaemic respiratory failure and gas exchange and radiological findings similar to those generally considered to be indications for invasive mechanical ventilation https://bit.ly/2GpBx6a

Cite this article as: Brusasco C, Corradi F, Di Domenico A, et al. Continuous positive airway pressure in COVID-19 patients with moderate-to-severe respiratory failure. Eur Respir J 2021; 57: 2002524 [https://doi. org/10.1183/13993003.02524-2020]. 
a)

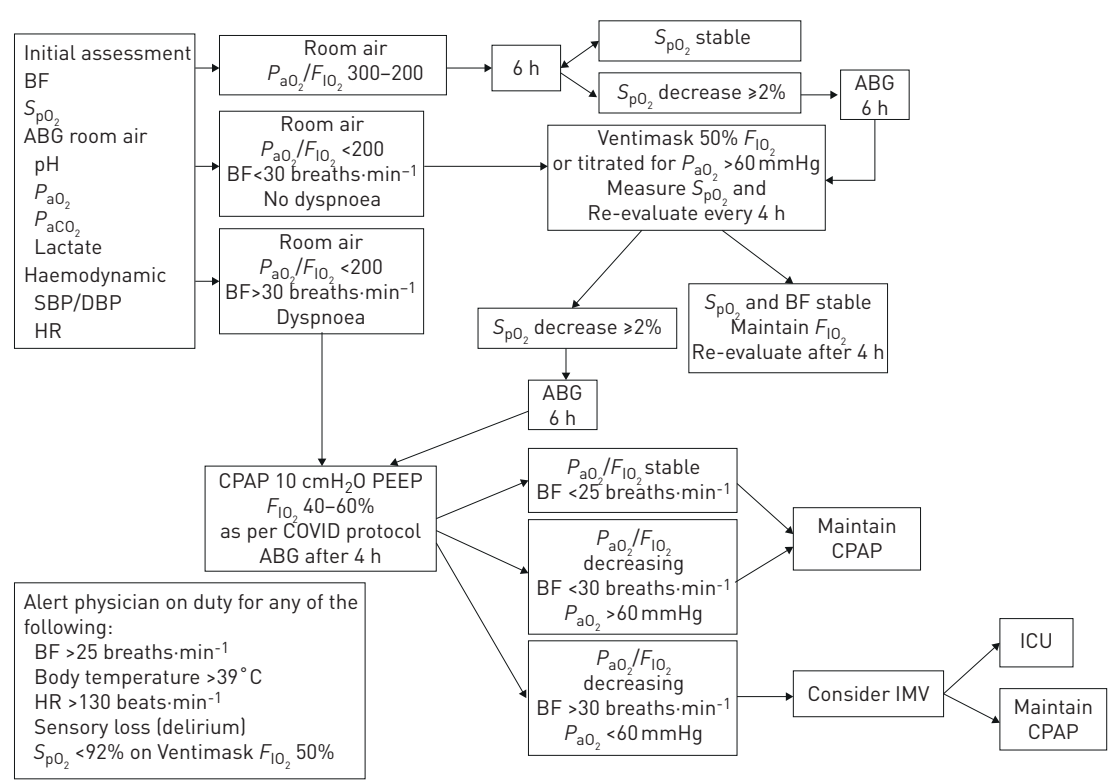

$\mathrm{PO}_{2}<92 \%$ on Ventimask $\mathrm{F}_{\mathrm{IO}_{2}} 50 \%$ b)

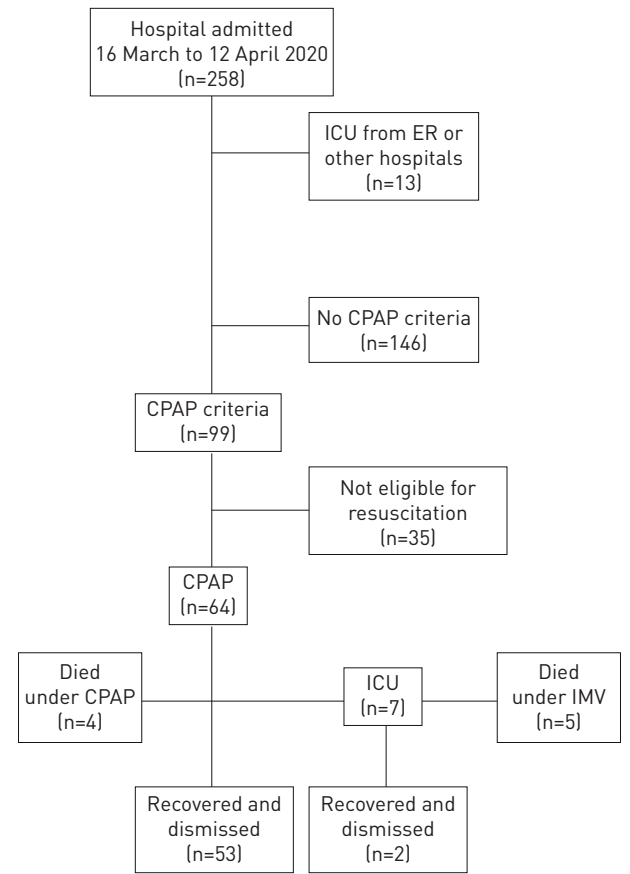

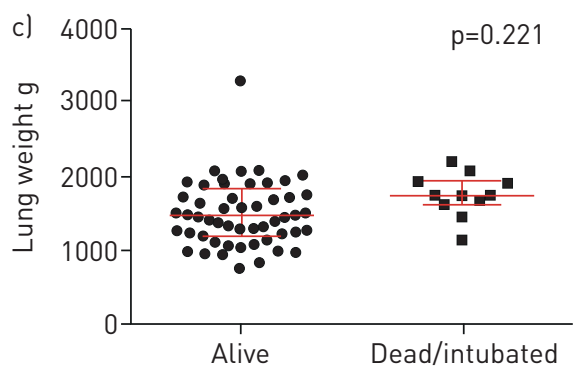

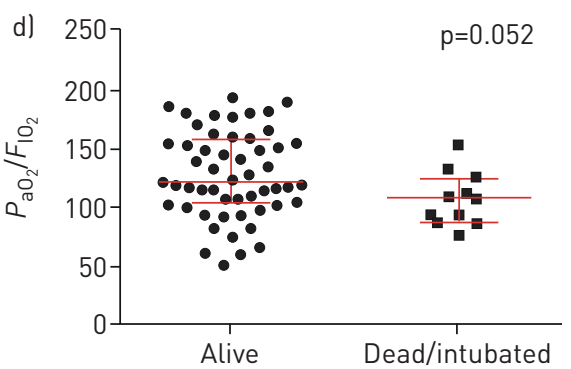

FIGURE 1 a) Algorithm for respiratory support given to coronavirus disease 2019 (COVID-19) patients. BF: breathing frequency; ABG: arterial blood gas; $\mathrm{S}_{\mathrm{pO}_{2}}$ : oxygen saturation measured by pulse oximetry; $P_{\mathrm{aO}_{2}} / F_{\mathrm{IO}_{2}}$ : arterial oxygen tension/inspiratory oxygen fraction ratio; $P_{\mathrm{acO}}$ : arterial carbon dioxide tension; SBP: systolic blood pressure; DBP: diastolic blood pressure; HR: heart rate; CPAP: continuous positive airway pressure; PEEP: positive end-expiratory pressure; IMV: invasive mechanical ventilation; ICU: intensive care unit. b) Study diagram. ER: emergency room. c, d) Scatterplots of individual values of computed tomography estimated c) lung weight and $\mathrm{c} / P_{\mathrm{aO}_{2}} / F_{\mathrm{IO}_{2}}$, with medians and interquartile ranges.

least two consecutive days. During this phase, Ventimask 50\% was used in the daytime and CPAP overnight. When morning and evening arterial blood gases were comparable, CPAP was ultimately withheld.

CT images (5.00-mm slice thickness) were analysed by ITK-snap software with semi-automatic and manual segmentation of lung parenchyma to calculate lung weight. Categorical variables were compared by Fisher exact test, continuous variables by Mann-Whitney U-test and presented as median (interquartile range; IQR). Factors associated with CPAP failure were first determined by univariate logistic regression analysis, including all anthropometric and clinical data. The variables achieving a $\mathrm{p}$-value $<0.1$ at univariate analysis were included in a multivariate model analysis. Statistical significance was assumed at two-tailed $\mathrm{p}<0.05$.

The total number of patients admitted to hospital with COVID-19 pneumonia over the period considered was 258 (figure 1b). 13 were directly admitted to the intensive care unit from emergency room or other hospitals, 181 were treated by Ventimask as per algorithm $(n=146)$ or because they were found to be ineligible for resuscitation at triage, due to extremely old age or life threatening comorbidities $(n=35)$. Thus, 64 patients underwent CPAP. They had an age range of 25-86 years and had been symptomatic for 7 (IQR 3-10) days. $P_{\mathrm{aO}_{2}} / F_{\mathrm{IO}_{2}}$ was 119 (IQR 99-153), breathing frequency was 33 (IQR 30-38) breaths $\mathrm{min}^{-1}$, lung attenuation >-750 $\mathrm{HU}$, and lung weight 1517 (IQR 1256-1870) g. 15 patients had been judged ineligible for resuscitation at triage but underwent CPAP thanks to device availability.

53 (83\%) patients recovered with CPAP within 17 days and were dismissed within 28 days. Four patients died under CPAP and seven required IMV. Three of the four patients who died under CPAP and one who died under IMV were among those considered ineligible for resuscitation at triage. 
At univariate analysis, odds for CPAP failure were male sex, hypertension, diabetes, COPD, having three comorbidities and lung weight. However, at multivariate analysis, only hypertension remained independently associated with CPAP failure (OR 7.33, 95\% CI 1.5-34, p=0.012). Baseline $P_{\mathrm{aO}_{2}} / F_{\mathrm{IO}_{2}}$ tended to be lower $(\mathrm{p}=0.052)$ in patients with CPAP failure (figure $1 \mathrm{c}$ and $\mathrm{d}$ ) but having $P_{\mathrm{aO}} / F_{\mathrm{IO}_{2}}<150$ was insignificant as a risk factor; 36 out of 53 patients recovering with CPAP had $P_{\mathrm{aO}_{2}} / F_{\mathrm{IO}_{2}}<150$. Lung weight was higher $(\mathrm{p}=0.031)$ in subjects with CPAP failure but having lung weight $>1.5 \mathrm{~kg}$ was insignificant as a risk factor; 23 of the 53 patients recovering with CPAP had lung weight $>1.5 \mathrm{~kg}$.

None of staff workers developed signs or symptoms of COVID-19 from the start of this study to the submission date.

Our main finding was that the vast majority of COVID-19 patients treated by CPAP recovered from moderate-to-severe AHRF, including cases with gas exchange and radiological findings similar to those considered as indications for IMV in typical adult respiratory distress syndrome (ARDS).

Since the outbreak of COVID-19, the majority of patients with moderate-to-severe AHRF have been treated by IMV, based on subjective judgement or criteria generally used for typical ARDS [8,9], with 49-60\% mortality [3-5]. Early observations in a few intubated patients led us to postulate that COVID-19 AHRF may differ from typical ARDS for relatively preserved respiratory mechanics despite similarly severe shunt $\left(P_{\mathrm{aO}_{2}} / F_{\mathrm{IO}_{2}}\right)$ and CT abnormalities [10]. Although this has been recently confuted [11], the possibility exists that IMV with high positive end-expiratory pressure may be unnecessary or even harmful in a number of COVID-19 patient and its liberal use should be considered with caution $[12,13]$. For the above reasons, we decided to use CPAP in all patients presenting with signs of severe intrapulmonary shunt $\left(P_{\mathrm{aO}_{2}} / F_{\mathrm{IO}_{2}}<200\right.$ or $P_{\mathrm{aO}_{2}}<60 \mathrm{mmHg}$ on Ventimask 50\%) or increased work of breathing (breathing frequency $>30$ breaths. $\min ^{-1}$ or dyspnoea) before considering IMV. In order to maintain the mechanical stress at a minimum and based on prior observations in intubated patients, we set CPAP at $10 \mathrm{cmH}_{2} \mathrm{O}$ in all patients; working with high airflow with the aim of reducing breathing frequency and dyspnoea at the cost of tolerating slight decrements of $P_{\mathrm{aO}_{2}} / F_{\mathrm{IO}_{2}}$.

The important results of our study are that neither $P_{\mathrm{aO}_{2}} / F_{\mathrm{IO}_{2}}$ nor lung weight were predictors of CPAP failure. In particular, CPAP avoided death or intubation in 36 out of 53 patients with $P_{\mathrm{aO}_{2}} / F_{\mathrm{IO}_{2}}<150$ and/ or lung weight $>1.5 \mathrm{~kg}$, which are usually considered as indications for IMV in typical ARDS [9]. In particular, $P_{\mathrm{aO}_{2}} / F_{\mathrm{IO}_{2}}<150$ was associated with higher mortality in a large multicentre study comparing NIV with IMV in typical ARDS, [14]. Although we also obtained favourable results with CPAP in patients meeting the criteria of severe ARDS, we cannot say whether this was due to COVID-19 AHRF differing from other forms of ARDS.

In COVID-19 AHRF, few studies reported occasional use of NIV [3, 4, 15] without giving technical details or selection criteria and only one considered the mortality of NIV separately from IMV without finding a statistically significant difference [5]. More recently, two research letters reported on CPAP. ORANGER et al. [6], using high-end home mechanical ventilators, found that CPAP avoided intubation in about $75 \%$ of patients, but there was no information to relate outcomes to severity of respiratory failure or CT imaging. AliBERTi et al. [7], using helmets with high-flow generators, found that CPAP avoided intubation in only about half of patients. A possible explanation for higher CPAP success in our study is a difference in criteria for intubation.

Limitations of the present study are the retrospective nature without comparator group, the single-centre and the small sample size, which limits the generalisability of results regarding risk factors and effects of pharmacological treatments.

In conclusion, our results suggest that a number of patients with moderate-to-severe AHRF due to COVID-19 pneumonia may be amenable to high-flow CPAP, even in the presence of gas exchange and CT findings usually considered as indications for IMV or even extracorporeal oxygenation in typical ARDS.

Claudia Brusasco ${ }^{1}$, Francesco Corradi $\circledast^{1,2}$, Antonia Di Domenico ${ }^{3}$, Francesca Raggi ${ }^{4}$, Graziano Timossi ${ }^{4}$, Gregorio Santori $^{5}$ and Vito Brusasco ${ }^{6}$, for the Galliera CPAP-Covid-19 study group ${ }^{7}$

${ }^{1}$ Anaesthesia and Intensive Care Unit, E.O. Ospedali Galliera, Genoa, Italy. ${ }^{2}$ Dept of Surgical, Medical, Molecular Pathology and Critical Care Medicine, University of Pisa, Pisa, Italy. ${ }^{3}$ Dept of Urology, E.O. Ospedali Galliera, Genoa, Italy. ${ }^{4}$ Dept of Internal Medicine, E.O. Ospedali Galliera, Genoa, Italy. ${ }^{5}$ Dept of Surgical Sciences and Integrated Diagnostics (DISC), University of Genoa, Genoa, Italy. ${ }^{6}$ Dept of Experimental Medicine, University of Genoa, Genoa, Italy. ${ }^{7} \mathrm{~A}$ list of collaborators from the Galliera CPAP-Covid-19 study group can be found in the Acknowledgements section.

Correspondence: Claudia Brusasco, Anaesthesia and Intensive Care Unit, E.O. Ospedali Galliera, Mura della Cappuccine 14, 16128 Genoa, Italy. E-mail: claudia.brusasco@gmail.com

Received: 09 June 2020 | Accepted: 28 Sept 2020

This article has an editorial commentary: https://doi.org/10.1183/13993003.04247-2020 
Author contributions: C. Brusasco and F. Corradi contributed to the study design, data collection and analysis, and the writing of the manuscript, and are the guarantors of the paper; F. Raggi and G. Timossi contributed to the data collection, data analysis, reading and checking of the manuscript; A. Di Domenico contributed as data manager, building of the database, data analysis, reading and checking of the manuscript; G. Santori contributed to statistical analysis, data results analysis, reading and checking of the manuscript; V. Brusasco contributed to the study design, data results interpretation and writing of the manuscript.

The collaborators of the Galliera CPAP-COVID-19 study group are: Giancarlo Antonucci, Elisabetta Blasi Vacca, Nicoletta Bobbio, Marcello Feasi, Francesco Forfori, Patrizia Guido, Marco Lattuada, Leila Mameli, Donatella Parodi, Roberta Pienovi, Emanuele Pontali, Maria Concetta Scirocco and Marina Terrile.

Conflict of interest: None declared.

\section{References}

1 Rochwerg B, Brochard L, Elliott MW, et al. Official ERS/ATS clinical practice guidelines: noninvasive ventilation for acute respiratory failure. Eur Respir J 2017; 50: 1602426.

2 Vitacca M, Nava S, Santus P, et al. Early consensus management for non-ICU ARF SARS-CoV-2 emergency in Italy: from ward to trenches. Eur Respir J 2020; 55: 2000632.

3 Grasselli G, Zangrillo A, Zanella A, et al. Baseline Characteristics and outcomes of 1591 patients infected with SARS-CoV-2 admitted to ICUs of the Lombardy Region, Italy. JAMA 2020; 323: 1574-1581.

4 Bhatraju PK, Ghassemieh BJ, Nichols M, et al. Covid-19 in critically ill patients in the Seattle region - case series. N Engl J Med 2020; 382: 2012-2022

5 Yang $\mathrm{X}, \mathrm{Yu} \mathrm{Y}, \mathrm{Xu} J$, et al. Clinical course and outcomes of critically ill patients with SARS-CoV-2 pneumonia in Wuhan, China: a single-centered, retrospective, observational study. Lancet Respir Med 2020; 8: 475-481.

6 Oranger M, Gonzalez-Bermejo J, Dacosta-Noble P, et al. Continuous positive airway pressure to avoid intubation in SARS-CoV-2 pneumonia: a two-period retrospective case-control study. Eur Respir J 2020; 56: 2001692.

7 Aliberti S, Radovanovic D, Billi F, et al. Helmet CPAP treatment in patients with COVID-19 pneumonia: a multicenter, cohort study. Eur Respir J 2020; 56: 2001935.

8 ARDS Definition Task Force, Ranieri VM, Rubenfeld GD, et al. Acute respiratory distress syndrome: the Berlin Definition. JAMA 2012; 307: 2526-2533.

9 Maiolo G, Collino F, Vasques F, et al. Reclassifying acute respiratory distress syndrome. Am J Respir Crit Care Med 2018; 197: 1586-1595.

10 Gattinoni L, Coppola S, Cressoni M, et al. Covid-19 does not lead to a "typical" acute respiratory distress syndrome. Am J Respir Crit Care Med 2020; 201: 1299-1300.

11 Bos LDJ, Sinha P, Dickson RP. Response to COVID-19 phenotyping correspondence. Eur Respir J 2020; 56: 2002756.

12 Tobin MJ, Laghi F, Jubran A. Caution about early intubation and mechanical ventilation in COVID-19. Ann Intensive Care 2020; 10: 78.

13 Tobin MJ. Basing respiratory management of coronavirus on physiological principles. Am J Respir Crit Care Med 2020; 201: 1319-1320.

14 Bellani G, Laffey JG, Pham T, et al. Noninvasive ventilation of patients with acute respiratory distress syndrome. Insights from the LUNG SAFE Study. Am J Respir Crit Care Med 2017; 195: 67-77.

15 Zhou F, Yu T, Du R, et al. Clinical course and risk factors for mortality of adult inpatients with COVID-19 in Wuhan, China: a retrospective cohort study. Lancet 2020; 395: 1054-1062. 